\title{
Optinalysis: Isometric Isomorphism and Automorphism Through A Looking-Glass
}

\author{
Kabir Bindawa Abdullahi \\ Department of Biology, Faculty of Natural and Applied Sciences, \\ Umaru Musa Yar'adua University, Katsina-Nigeria \\ *Corresponding author's email: Kabir.abdullahi@umyu.edu.ng; kabirnamallam@gmail.com
}

\begin{abstract}
Measures of graph symmetry, similarity, and identity have been extensively studied in graph automorphism and isomorphism detection problems. Nevertheless, graph isomorphism detection remains an open (unsolved) problem for many decades. In this paper, a new and efficient methodological paradigm, called optinalysis, is proposed for symmetry detections, similarity, and identity measures between isometric isomorphs or automorphs. Optinalysis is explained and expressed in clearly stated definitions and prove theorems, which conform to the definitions and theorems of isometry, isomorphism, and automorphism. Analogous to the polynomiality formalization for an efficient algorithm for graph isomorphism detection, optinalysis is however deterministic on polynomial and non-polynomial graph models.
\end{abstract}

Keywords: Automorphism; Identity; Isometry; Isomorphism; Similarity; Symmetry.

\section{Introduction}

The notion of isometry (as a congruence mapping) is a general phenomenon commonly accepted in Mathematics. It means a mapping that preserves distances. It is a bijective mapping, characterized as one-to-one mapping of a group onto itself or onto another in various transformational ways such as reflections, translation, or rotations (Miillman, R and Parker, G 1981).

Two graphs or sets are isomorphic if there is a bijection between their vertices or elements that preserves adjacency; such a bijection is called an isomorphism. In other terms, two graphs or sets $A$ and $B$ as isomorphic if they have the same structure, but their elements or vertices may be different. An isomorphism from a graph onto itself is called an automorphism, and the set of all automorphisms of a given graph $G$ denoted $\operatorname{Aut}(G)$, forms a group under composition (Hatori, Miura, and Takagi 2006).

The graph isomorphism problem is that of detecting the existence (or not) of an isomorphism between two input graphs. It is among a notorious problem in complexity theory, as no polynomial-time algorithm is known, and at the same time graph isomorphism is generally not believed to be NP-complete (Laszlo 2016). Babai recently presented a quasipolynomial time algorithm for graph isomorphism (Oded, Silvio, and Wigderson 1991). It is well-known that graph isomorphism is algorithmically and computationally polynomial-time equivalent (Ronald, C, and Derek, G 1977; Mathon 1979).

In applications, graph isomorphism has been applied to many structural entities from molecules to objects, and from music to physics, etc. For instance, several methods for detection of isomorphism problems in Kinematics such as Linkage Characteristic Polynomials Method, Hamming Number Technique, Degree Code Method, Link Adjacency Table Method, Distance Concept, Neural Network Approach, Fuzzy Logic Approach, Loop Based Detection Method, Genetic Algorithm Approach, Spanning Tree Method, Adjacency Matrix Method, Joint-Joint Matrix Method, etc have been proposed by many scholars from machine learning and algorithmic community (Arora \& Nigam, S, 2013; Shane et al., 
2014). In addition to the polynomiality formalization, reliability of results, simplicity, applicability, detection of inversions, etc are some of the definable attributes of efficient methods or algorithms in graph isomorphism detections (Arora \& Nigam, S, 2013; Shane et al., 2014). Until date, none of the existing methods is generally accepted to be efficient and a complete solution.

In this paper, optinalysis is proposed which looks at two finite graphs and sets as isometric isomorphs or automorphs as a mirror-like reflection of each other that expresses their degree of symmetry or identity and similarity. Optinalysis is expressed in clearly defined terms and proven theorems, which conforms to the definitions and theorems of isometry, isomorphism, and automorphism.

\section{Preliminary definitions and theorems}

Definition I. Injections, surjections, and bijections of functions between sets.

These are words that describe certain functions $f: A \rightarrow B \rightarrow$ Bfrom one set to another.

An injection, also called a one-to-one function is a function that maps distinct elements to distinct elements, that is, if $\mathrm{x} \neq \mathrm{y}$, then $\mathrm{f}(\mathrm{x}) \neq \mathrm{f}(\mathrm{y})$. Equivalently, if $\mathrm{f}(\mathrm{x})=\mathrm{f}(\mathrm{y})$ then, $\mathrm{x}=\mathrm{y}$.

$A$ surjection also called an onto function is one that includes all of $B$ in its image, that is, if $y \in B$, then there is an $x \in A$ such that $f(x)=y$.

A bijection, also called a one-to-one correspondence, is a function that is simultaneously injective and bijective. Another way to describe a bijection $f: A \rightarrow B$ is to say that there is an inverse function $g$ : $B \rightarrow A$ so that the composition $g$ of $: A \rightarrow A$ is the identity function on $A$ while $f$ o $g: B \rightarrow B$ is the identity function on $B$. The usual notation for the function inverse to $f$ is $f^{-1}$.

If $f$ and $g$ are inverse to each other, that is, if $g$ is the inverse of $f, g=f^{-1}$, then $f$ is the inverse of $g, f=g^{-1}$ Thus, $\left(f^{-1}\right)^{-1}=f$.

An important property of bijections is that you can convert equations involving $f$ to equations involving $f^{-1}$ :

$$
f(x)=y \text { if and only if } x=f^{-1}(y) .
$$

Definition II. Isometry (or congruence or congruent transformation) is a distance-preserving transformation between metric spaces, usually assumed to be bijective. Let $A$ and $B$ be metric space with metrics $d_{A}$ and $d_{B}$. A map $f: A \rightarrow B$ is called an isometry or distance preserving if for any $a, b \in A$ one has

$$
d_{B}\left(f(a), f(b)=d_{A}(a, b)\right.
$$

Definition III. Isomorphism is a vertex bijection that preserves the mathematical structures (e.g, vertices, edges, and non-edges) between two spaces sets and graphs that can be reversed by inverse mapping. Two mathematical structures $A$ and $B$ are isomorphic if they have the same structure, but their elements may be different.

$$
\begin{gathered}
f: A \rightarrow B \\
A \cong B
\end{gathered}
$$

Definition IV. Automorphism is an isomorphism from a mathematical object to itself. It is, in some sense; define the symmetry of the object, and a way of mapping the object to itself while preserving all of its mathematical structure (e.g vertices, edges, and non-edges). 


$$
\begin{gathered}
f: A \rightarrow \operatorname{Aut}\left(A^{\prime}\right) \\
A \cong A^{\prime}
\end{gathered}
$$

Definition $\boldsymbol{V}$. Scale can be defined as the system of marks at fixed intervals, which define the relationship between the units being used and their representation on the graph.

Theorem I. An isometry maps

(i) straight lines to straight lines;

(ii) segments to congruent segments;

(iii) triangles to congruent triangles;

(iv) angles to congruent angles.

Theorem II. Any isometry of the plane is a composition of at most three reflections.

Theorem III. A symmetry about a point is an isometry.

\section{Definitions and Theorems of Optinalysis}

\subsection{Definitions}

\section{Definition 1:}

Optinalysis is a function that automorphically or isomorphically compares the symmetry, similarity, and identity between two spaces, graphs, and sets as a mirror-like (optic-like) reflection of each other about a symmetrical line or mid-point.

\section{Definition 2:}

Optinalysis is a function that comprised of an assigned optical (mirror) scale $(R)$ that bijectively re-maps $(\rightarrow)$ isometric and isomorphic or automorphic spaces, sets, and graphs under reflection about a symmetrical line or mid-point $(\delta)$. Figure 1 illustrates how two isometric isomorphs or automorphs are mapped and also re-mapped by an optical scale.

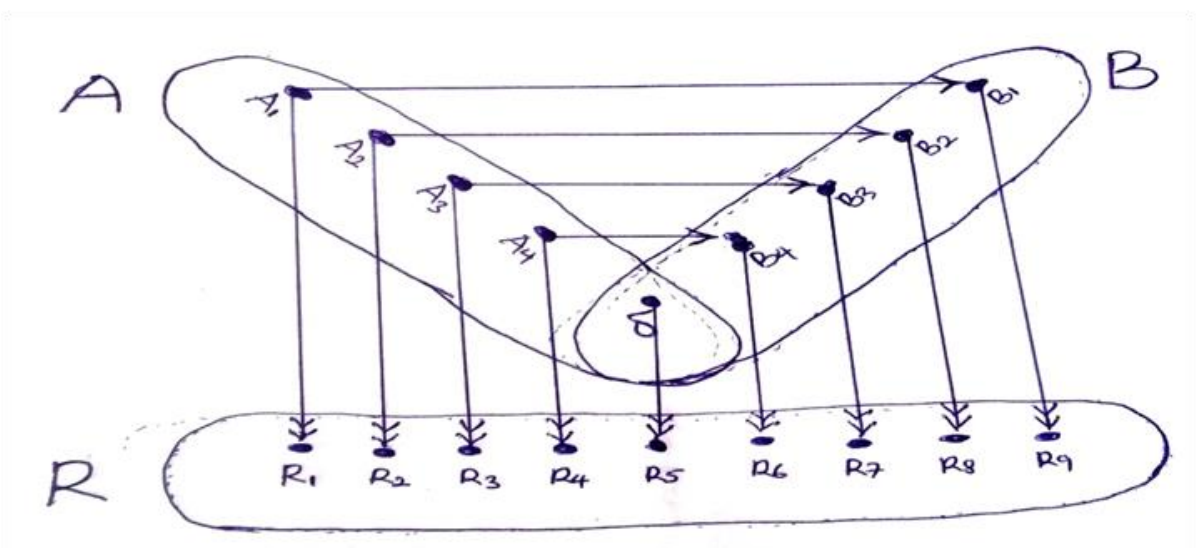

Figure I: Mapping between two isometric isomorphs or automorphs and re-mapping with the optical scale. $A$ is a domain; $B$ is a co-domain; $\delta$ is a mid-point or symmetrical line, and $R$ is an optical scale. The symbol $\rightarrow$ indicates a bijective mapping between the isometric isomorphs around a centre (midpoint) and $\rightarrow$ indicates a bijective re-mapping by the optical scale. 
Optinalysis is expressed or defined by optinalytic construction. An optinalytic construction is the mathematical representation of optinalysis between two isometric isomorphs or automorphs.

Optinalysis is defined in two broad types: shape (automorphic or intrametric) and comparative (isomorphic or intermetric) optinalysis.

\section{Definition 2.1: Shape (Automorphic or Intrametric) Optinalysis:}

Shape or automorphic or intrametric optinalysis is an optinalysis of isometric automorphs (bijective mapping of a set onto itself). It is a method of symmetry detection. Shape optinalysis is defined by its optinalytic constructions as:

$$
\begin{aligned}
& f: A \underset{\Pi}{\delta} A^{\prime} \rightarrow R \\
& f: A=\left(A_{1}, A_{2}, A_{3}, \ldots \ldots, A_{n}\right) \quad \underset{m}{\delta} A^{\prime}=\left(A^{\prime}{ }_{n}, \ldots \ldots, A^{\prime}{ }_{3}, A^{\prime}{ }_{2}, A^{\prime}{ }_{1}\right) \rightarrow R=\left( \pm R_{1}, \pm R_{2}, \pm R_{3}, \ldots \ldots, \pm R_{n}\right) \\
& f:\left[\begin{array}{ccc}
A=\left(A_{1}, A_{2}, A_{3}\right) & \delta & A^{\prime}=\left(A^{\prime}{ }_{3}, A^{\prime}{ }_{2}, A^{\prime}{ }_{1}\right) \\
\Downarrow & \stackrel{\#}{*} & \Downarrow \\
R=\left( \pm R_{1}, \pm R_{2}, \pm R_{3}\right) & R_{4} & \left( \pm R_{5}, \pm R_{6}, \pm R_{7}\right)
\end{array}\right]
\end{aligned}
$$

Such that: $\left(A_{1}, A_{2}, A_{3}\right) \in A ;\left(A^{\prime}{ }_{1}, A^{\prime}{ }_{2}, A^{\prime}{ }_{3}\right) \in A^{\prime} ; \delta \in A \& A^{\prime} ; A, A^{\prime \prime} \& R \in \mathbb{R}, \mathbb{R}^{\mathrm{m}} ; R_{1} \neq 0 ;$ and $A \& B$ are isometric automorphs of a named space, sets, and graphs.

\section{Definition 2.2: Comparative (Isomorphic or Intermetric) Optinalysis:}

Comparative or isomorphic or intermetric optinalysis is an optinalysis of isometric isomorphs (bijective mapping of a set onto others). It is a method of similarity and identity measures. Comparative optinalysis is defined by its optinalytic constructions as:

$$
\begin{aligned}
& f: A \underset{\mathrm{m}}{\delta} \quad B \rightarrow R
\end{aligned}
$$

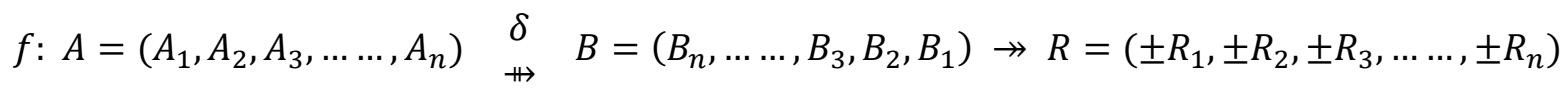

$$
\begin{aligned}
& f:\left[\begin{array}{ccc}
A=\left(A_{1}, A_{2}, A_{3}\right) & \delta & B=\left(B_{3}, B_{2}, B_{1}\right) \\
\downarrow & \stackrel{\Pi}{ } & \downarrow \\
R=\left( \pm R_{1}, \pm R_{2}, \pm R_{3}\right) & \pm R_{4} & \left( \pm R_{5}, \pm R_{6}, \pm R_{7}\right)
\end{array}\right]
\end{aligned}
$$

Such that: $\left(A_{1}, A_{2}, A_{3}\right) \in A ;\left(B_{1}, B_{2}, B_{3}\right) \in B ; \delta \notin A \& B ; A, B \& R \in \mathbb{R}, \mathbb{R}^{\mathrm{m}} ; R_{1} \neq 0 ;$ and $A \& B$ are isometric isomorphs of a named space, sets, and graphs.

\section{Definition 2.3: Head-to-head Reflection or Pairing}

In comparative optinalysis, a reflection (pairing) is said to be head-to-head if the first labeled elements of two graphs, sets are the terminal points of the reflecting isometric isomorphs.

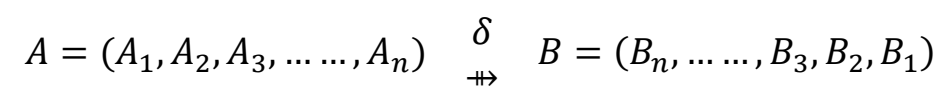




\section{Definition 2.4: Tail-to-tail Reflection or Pairing}

In comparative optinalysis, a reflection or pairing is said to be tail-to-tail if the last labeled elements of graphs, sets are the terminal point of the reflecting isometric isomorphs.

$$
A=\left(A_{n}, \ldots \ldots, A_{3}, A_{2}, A_{1}\right) \underset{\leftrightarrow}{\delta} \quad B=\left(B_{1}, B_{2}, B_{3}, \ldots \ldots, B_{n}\right)
$$

\section{Definition 3:}

Optinalysis is a function that bijectively maps optical nodes $(N)$ to their corresponding optical nodes $(N)$ about a symmetrical line or mid-point $(\delta)$. An optical node is defined by its scalement. A scalement, is the product of any member of a set and its assigned optical scale.

Construction by Shape Optinalysis

Suppose we have an optinalytic construction of isometric and automorphic spaces, sets and graphs with an assigned optical scale $(\mathrm{R}=1,2,3,4,5,6,7)$ as follows:

$$
f:\left[\begin{array}{ccc}
A=\left(A_{1}, A_{2}, A_{3}\right) & \delta & A^{\prime}=\left(A^{\prime}{ }_{3}, A^{\prime}{ }_{2}, A^{\prime}{ }_{1}\right) \\
\Downarrow & \Downarrow & \Downarrow \\
R=(1,2,3) & 4 & (5,6,7)
\end{array}\right]
$$

Such that $A$ and $A^{\prime}$ are isometric isomorphs on head-to-head reflection about a centre $(\delta)$.

$$
\begin{aligned}
& f: N \underset{\rightarrow}{\delta} \quad N^{\prime} \\
& N=A \times R, \quad \& \quad N^{\prime}=A^{\prime} \times R \\
& f: A \times R \quad \underset{\Pi}{\delta} \quad A^{\prime} \times R \\
& f: A=\left(A_{1}, 2 A_{2}, 3 A_{3}\right) \quad \underset{\#}{4} \quad A^{\prime}=\left(5 A^{\prime}{ }_{3}, 6 A^{\prime}{ }_{2}, 7 A_{1}^{\prime}\right)
\end{aligned}
$$

\section{Construction by Comparative Optinalysis}

Suppose we have an optinalytic construction of isometric and isomorphic spaces, sets and graphs with an assigned optical scale $(\mathrm{R}=1,2,3,4,5,6,7)$ as follows:

$$
f:\left[\begin{array}{ccc}
A=\left(A_{1}, A_{2}, A_{3}\right) & \delta & B=\left(B_{3}, B_{2}, B_{1}\right) \\
\Downarrow & \sharp & \Downarrow \\
R=(1,2,3) & 4 & (5,6,7)
\end{array}\right]
$$

Such that $A$ and $B$ are isometric isomorphs on head-to-head reflection about a centre $(\delta)$.).

$$
\begin{aligned}
& f: N \underset{\rightarrow}{\delta} N^{\prime} \\
& N=A \times R \quad \& \quad N^{\prime}=B \times R
\end{aligned}
$$

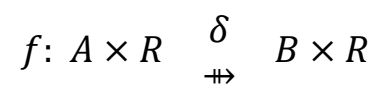




$$
f: A=\left(A_{1}, 2 A_{2}, 3 A_{3}\right) \underset{\rightarrow}{4 \delta} \quad B=\left(5 B_{3}, 6 B_{2}, 7 B_{1}\right)
$$

\subsection{Theorems}

\section{Theorem 1: Kabirian coefficient of optinalysis}

Isometric and isomorphic or automorphic spaces, sets and graphs are symmetrical or identical and similar to a certain magnitude by a coefficient, called Kabirian coefficient (denoted as $\left(K_{c}\right)$.

\section{Definition 4:}

Kabirian coefficient is expressed as the divisible product of the median optical scale $\left(\frac{N_{d}+1}{2}\right.$, where $N_{d}$ is the total count of the established nodes or vertices, this is called nodality) and the sum of all members (elements) by the sum of all scalements (optical nodes).

\section{Prove 1:}

Construction by Shape Optinalysis

Suppose we have an optinalytic construction of isometric and automorphic spaces, sets and graphs with an assigned optical scale $(\mathrm{R}=1,2,3,4,5,6,7)$ as follows:

$$
f:\left[\begin{array}{ccc}
A=\left(A_{1}, A_{2}, A_{3}\right) & \delta & A^{\prime}=\left(A^{\prime}{ }_{3}, A^{\prime}{ }_{2}, A^{\prime}{ }_{1}\right) \\
\Downarrow & \Downarrow & \Downarrow \\
R=(1,2,3) & 4 & (5,6,7)
\end{array}\right]
$$

Such that $A$ and $A^{\prime}$ are isometric automorphs on head-to-head reflection about a centre $(\delta)$.

Then, the Kabirian coefficient of symmetry or identity and similarity between the two isometric isomorphs is expressed as (Equations 1):

$$
K_{c}=\frac{4\left(A_{1}+A_{2}+A_{3}+\delta+A_{3}^{\prime}+A_{2}^{\prime}+A_{1}^{\prime}\right)}{\left(A_{1}+2 A_{2}+3 A_{3}+4 \delta+5 A^{\prime}{ }_{3}+6 A_{2}^{\prime}+7 A^{\prime}\right)}
$$

Such that each element is expressed as (Equations 1.1-1.7):

$$
\begin{aligned}
& A_{1}=\frac{K_{c}\left(2 A_{2}+3 A_{3}+4 \delta+5 A^{\prime}{ }_{3}+6 A^{\prime}{ }_{2}+7 A^{\prime}{ }_{1}\right)-4\left(A_{2}+A_{3}+\delta+A^{\prime}{ }_{3}+A^{\prime}{ }_{2}+A^{\prime}{ }_{1}\right)}{4-K_{c}} \\
& A_{2}=\frac{K_{c}\left(A_{1}+3 A_{3}+4 \delta+5 A_{3}^{\prime}+6 A^{\prime}{ }_{2}+7 A_{1}^{\prime}\right)-4\left(A_{1}+A_{3}+\delta+A^{\prime}{ }_{3}+A^{\prime}{ }_{2}+A_{1}^{\prime}{ }_{1}\right)}{4-2 K_{C}} \\
& A_{3}=\frac{K_{c}\left(A_{1}+2 A_{2}+4 \delta+5 A^{\prime}{ }_{3}+6 A_{2}^{\prime}+7 A_{1}^{\prime}\right)-4\left(A_{1}+A_{2}+\delta+A^{\prime}{ }_{3}+A_{2}^{\prime}+A^{\prime}{ }_{1}\right)}{4-3 K_{c}} \\
& \delta=\frac{K_{c}\left(A_{1}+2 A_{2}+3 A_{3}+5 A^{\prime}{ }_{3}+6 A^{\prime}{ }_{2}+7 A^{\prime}{ }_{1}\right)-4\left(A_{1}+A_{2}+A_{3}+A^{\prime}{ }_{3}+A^{\prime}{ }_{2}+A^{\prime}{ }_{1}\right)}{4-4 K_{c}} \\
& A^{\prime}{ }_{3}=\frac{K_{c}\left(A_{1}+2 A_{2}+3 A_{3}+4 \delta+6 A_{2}^{\prime}+7 A_{1}^{\prime}\right)-4\left(A_{1}+A_{2}+A_{3}+\delta+A_{2}^{\prime}+A_{1}^{\prime}\right)}{4-5 K_{c}}
\end{aligned}
$$




$$
\begin{aligned}
& A^{\prime}{ }_{2}=\frac{K_{c}\left(A_{1}+2 A_{2}+3 A_{3}+4 \delta+5 A^{\prime}{ }_{3}+7 A_{1}^{\prime}\right)-4\left(A_{1}+A_{2}+A_{3}+\delta+A^{\prime}{ }_{3}+A^{\prime}{ }_{1}\right)}{4-6 K_{C}} \\
& A^{\prime}{ }_{1}=\frac{K_{c}\left(A_{1}+2 A_{2}+3 A_{3}+4 \delta+5 A^{\prime}{ }_{3}+6 A^{\prime}{ }_{2}\right)-4\left(A_{1}+A_{2}+A_{3}+\delta+A^{\prime}{ }_{3}+A_{2}{ }_{2}\right)}{4-7 K_{c}}
\end{aligned}
$$

Construction by Comparative Optinalysis

Suppose we have an optinalytic construction of isometric and isomorphic spaces, sets and graphs with an assigned optical scale $(\mathrm{R}=1,2,3,4,5,6,7)$ as follows:

$$
f:\left[\begin{array}{ccc}
A=\left(A_{1}, A_{2}, A_{3}\right) & \delta & B=\left(B_{3}, B_{2}, B_{1}\right) \\
\Downarrow & \ddagger & \Downarrow \\
R=(1,2,3) & 4 & (5,6,7)
\end{array}\right]
$$

Such that $A$ and $B$ are isometric isomorphs on head-to-head reflection about a centre $(\delta)$.

Then, the Kabirian coefficient of symmetry or identity and similarity between the two isometric isomorphs is expressed as (Equations 2):

$$
K_{c}=\frac{4\left(A_{1}+A_{2}+A_{3}+\delta+B_{3}+B_{2}+B_{1}\right)}{\left(A_{1}+2 A_{2}+3 A_{3}+4 \delta+5 B_{3}+6 B_{2}+7 B_{1}\right)}
$$

Such that each element is expressed as (Equations 2.1-2.7):

$$
\begin{aligned}
& A_{1}=\frac{K_{c}\left(2 A_{2}+3 A_{3}+4 \delta+5 B_{3}+6 B_{2}+7 B_{1}\right)-4\left(A_{2}+A_{3}+\delta+B_{3}+B_{2}+B_{1}\right)}{4-K_{c}} \\
& A_{2}=\frac{K_{c}\left(A_{1}+3 A_{3}+4 \delta+5 B_{3}+6 B_{2}+7 B_{1}\right)-4\left(A_{1}+A_{3}+\delta+B_{3}+B_{2}+B_{1}\right)}{4-2 K_{c}} \\
& A_{3}=\frac{K_{c}\left(A_{1}+2 A_{2}+4 \delta+5 B_{3}+6 B_{2}+7 B_{1}\right)-4\left(A_{1}+A_{2}+\delta+B_{3}+B_{2}+B_{1}\right)}{4-3 K_{c}} \\
& \delta=\frac{K_{c}\left(A_{1}+2 A_{2}+3 A_{3}+5 B_{3}+6 B_{2}+7 B_{1}\right)-4\left(A_{1}+A_{2}+A_{3}+B_{3}+B_{2}+B_{1}\right)}{4-4 K_{c}} \\
& B_{3}=\frac{K_{c}\left(A_{1}+2 A_{2}+3 A_{3}+4 \delta+6 B_{2}+7 B_{1}\right)-4\left(A_{1}+A_{2}+A_{3}+\delta+B_{2}+B_{1}\right)}{4-5 K_{c}} \\
& B_{2}=\frac{K_{c}\left(A_{1}+2 A_{2}+3 A_{3}+4 \delta+5 B_{3}+7 B_{1}\right)-4\left(A_{1}+A_{2}+A_{3}+\delta+B_{3}+B_{1}\right)}{4-6 K_{c}} \\
& B_{1}=\frac{K_{c}\left(A_{1}+2 A_{2}+3 A_{3}+4 \delta+5 B_{3}+6 B_{2}\right)-4\left(A_{1}+A_{2}+A_{3}+\delta+B_{3}+B_{2}\right)}{4-7 K_{c}}
\end{aligned}
$$

\section{Lemma 1:}



functionally.

Pair of corresponding vertices under optinalysis is bijective (one-to-one and onto) to each other

\section{Prove:}

Recall the definition injective mapping (one-to-one), if $x=y$, then $f(x)=f(y)$, or equivalently if $x \neq y$, then $f(x) \neq f(y)$. Based on our problem under evaluation, and about equations 1.2 to 1.7 , and 2.1 to 2.7 of theorem 1 , we now have

Automorphically and isomorphically:

if $A_{1}=A^{\prime}{ }_{1}$, then $f\left(A_{1}\right)=f\left(A^{\prime}{ }_{1}\right)$ or equivalently, if $A_{1} \neq A^{\prime}{ }_{1}$, then $f\left(A_{1}\right) \neq f\left(B_{1}\right)$

if $A_{2}=A^{\prime}{ }_{2}$, then $f\left(A_{2}\right)=f\left(A^{\prime}{ }_{2}\right)$ or equivalently, if $A_{2} \neq A^{\prime}{ }_{2}$, then $f\left(A_{2}\right) \neq f\left(B_{2}\right)$

if $A_{3}=A^{\prime}{ }_{3}$, then $f\left(A_{3}\right)=f\left(A^{\prime}{ }_{3}\right)$ or equivalently, if $A_{3} \neq A_{3}^{\prime}$, then $f\left(A_{3}\right) \neq f\left(B_{3}\right)$

Recall the definition surjective mapping (onto itself or other), if $y \in A$, then there is an $x \in A$ such that $f(x)=y$. Based on our problem under evaluation, and about equations 1.2 to 1.7 , and 2.1 to 2.7 of theorem 1, we now have:

Isomorphically:

if $A_{1} \in A$, then there is an $A_{1}^{\prime} \in A$ such that $f\left(A_{1}\right)=A_{1}^{\prime}$

if $A_{2} \in A$, then there is an $A_{2}^{\prime} \in A$ such that $f\left(A_{2}\right)=A^{\prime}{ }_{2}$

if $A_{3} \in A$, then there is an $A_{3}^{\prime} \in A$ such that $f\left(A_{3}\right)=A_{3}^{\prime}$

Isomorphically and equivalently:

if $A_{1} \in A$, then there is an $B_{1} \in B$ such that $f\left(A_{1}\right)=B_{1}$

if $A_{2} \in A$, then there is an $B_{2} \in B$ such that $f\left(A_{2}\right)=B_{2}$

if $A_{3} \in A$, then there is an $B_{3} \in B$ such that $f\left(A_{3}\right)=B_{3}$

Therefore, optinalysis is based on the paradigm of the bijective mapping of defined finite sets of isometric isomorphs or automorphs.

\section{Theorem 2: Optinalytic invariance under operation (I)}

A perfect symmetry or identity and similarity state between isometric and isomorphic groups remains invariant (stable) with $K_{c}=1(100 \%)$ under other transformations such as pericentral rotation (alternate reflection), central rotation (inversion), product translation, additive translation, optical scaling, and central modulation.

\section{Prove 2:}

Suppose we have an optinalytic construction of isometric and isomorphic spaces, sets and graphs with an assigned optical scale $(\mathrm{R}=1,2,3,4,5,6,7)$ as follows: 


$$
f:\left[\begin{array}{ccc}
A=\left(x^{2}, x^{4}, x^{3}\right) & \delta & B=\left(x^{3}, x^{4}, x^{2}\right) \\
\Downarrow & \stackrel{\leftrightarrow}{\ddagger} & \Downarrow \\
R=(1,2,3) & 4 & (5,6,7)
\end{array}\right]
$$

Such that $A$ and $B$ are isometric isomorphs on head-to-head reflection about a centre $(\delta)$.

Then,

$$
\begin{gathered}
K_{c}=\frac{4\left(x^{2}+x^{4}+x^{3}+\delta+x^{3}+x^{4}+x^{2}\right)}{x^{2}+2 x^{4}+3 x^{3}+4 \delta+5 x^{3}+6 x^{4}+7 x^{2}} \\
K_{c}=\frac{4 x^{2}+4 x^{4}+4 x^{3}+4 \delta+4 x^{3}+4 x^{4}+4 x^{2}}{x^{2}+2 x^{4}+3 x^{3}+4 \delta+5 x^{3}+6 x^{4}+7 x^{2}} \\
K_{c}=\frac{8 x^{2}+8 x^{3}+8 x^{4}+4 \delta}{8 x^{2}+8 x^{3}+8 x^{4}+4 \delta}=1
\end{gathered}
$$

Therefore, $A$ and $B$ are perfectly similar and identical.

\section{Prove 2.1: Invariance under additive translation}

Let the optinalytic construction of prove 3 be considered, and let ' $a$ ' be a translation factor. The optinalytic construction becomes:

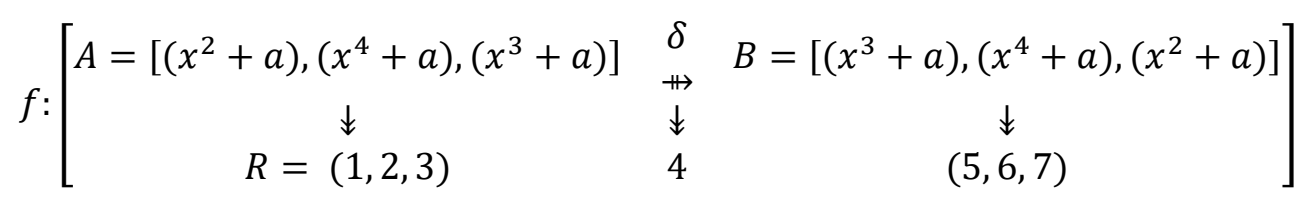

Such that $A$ and $B$ are isometric isomorphs on head-to-head reflection about a centre $(\delta)$.

Then,

$$
\begin{gathered}
K_{c}=\frac{4\left[\left(x^{2}+a\right)+\left(x^{4}+a\right)+\left(x^{3}+a\right)+\delta+\left(x^{3}+a\right)+\left(x^{4}+a\right)+\left(x^{2}+a\right)\right]}{\left(x^{2}+a\right)+2\left(x^{4}+a\right)+3\left(x^{3}+a\right)+4 \delta+5\left(x^{3}+a\right)+6\left(x^{4}+a\right)+7\left(x^{2}+a\right)} \\
K_{c}=\frac{8\left(x^{2}+a\right)+8\left(x^{4}+a\right)+8\left(x^{3}+a\right)+4 \delta}{8\left(x^{2}+a\right)+8\left(x^{4}+a\right)+8\left(x^{3}+a\right)+4 \delta}=1
\end{gathered}
$$

Therefore, $A$ and $B$ are invariant under translation.

\section{Prove 2.2: Invariance under product translation}

Let the optinalytic construction of prove 3 be considered, and let ' $a$ ' be a translation factor. The optinalytic construction becomes:

$$
f:\left[\begin{array}{ccc}
A=\left(a x^{2}, a x^{4}, a x^{3}\right) & \delta & B=\left(a x^{3}, a x^{4}, a x^{2}\right) \\
\Downarrow & \stackrel{\Pi}{*} & \Downarrow \\
R=(1,2,3) & 4 & (5,6,7)
\end{array}\right]
$$

Such that $A$ and $B$ are isometric isomorphs on head-to-head reflection about a centre $(\delta)$. 
Then,

$$
\begin{gathered}
K_{c}=\frac{4\left(a x^{2}+a x^{4}+a x^{3}+\delta+a x^{3}+a x^{4}+a x^{2}\right)}{a x^{2}+2 a x^{4}+3 a x^{3}+4 \delta+5 a x^{3}+6 a x^{4}+7 a x^{2}} \\
K_{c}=\frac{4 a x^{2}+4 a x^{4}+4 a x^{3}+4 \delta+4 a x^{3}+4 a x^{4}+4 a x^{2}}{a x^{2}+2 a x^{4}+3 a x^{3}+4 \delta+5 a x^{3}+6 a x^{4}+7 a x^{2}}=1 \\
K_{c}=\frac{8 a x^{2}+8 a x^{4}+8 a x^{3}+4 \delta}{8 a x^{2}+8 a x^{4}+8 a x^{3}+4 \delta}=1
\end{gathered}
$$

Therefore, $A$ and $B$ are invariant under translation.

\section{Prove 2.3: Invariance under central rotation (Inversion)}

Definition 5: Central rotation refers to the rotation of all the elements or vertices of the two isometric and isomorphic or automorphic spaces, sets, and graphs $180^{\circ}$ around the central mid-point $(\delta)$. This rotation is equivalent to an inversion. becomes:

Let the optinalytic construction of prove 3 be considered, the centrally rotated structures

$$
f:\left[\begin{array}{ccc}
B=\left(x^{2}, x^{4}, x^{3}\right) & \delta & A=\left(x^{3}, x^{4}, x^{2}\right) \\
\Downarrow & \stackrel{m}{ } & \Downarrow \\
R=(1,2,3) & 4 & (5,6,7)
\end{array}\right]
$$

Such that $A$ and $B$ are isometric isomorphs on head-to-head reflection about a centre $(\delta)$.

Then,

$$
\begin{gathered}
K_{c}=\frac{4\left(x^{2}+x^{4}+x^{3}+\delta+x^{3}+x^{4}+x^{2}\right)}{x^{2}+2 x^{4}+3 x^{3}+4 \delta+5 x^{3}+6 x^{4}+7 x^{2}} \\
K_{c}=\frac{4 x^{2}+4 x^{4}+4 x^{3}+4 \delta+4 x^{3}+4 x^{4}+4 x^{2}}{x^{2}+2 x^{4}+3 x^{3}+4 \delta+5 x^{3}+6 x^{4}+7 x^{2}} \\
K_{c}=\frac{8 x^{2}+8 x^{3}+8 x^{4}+4 \delta}{8 x^{2}+8 x^{3}+8 x^{4}+4 \delta}=1
\end{gathered}
$$

Therefore, $A$ and $B$ are invariant under central rotation.

\section{Prove 2.4: Invariance under pericentral rotation (Alternate reflection)}

Definition 6: Central rotation refers to as the rotation of all the elements or vertices of the isometric and isomorphic or automorphic spaces, sets and graphs $180^{\circ}$ around the pericentres (A pericentre is a midpoint of each of the two comparing structures). This structure is the same as the alternate reflection (i.e, the tail-to-tail reflection). An alternate reflection is the other alternative form of reflection between two reflecting isometric and isomorphic groups.

Let the optinalytic construction of prove 3 be considered, the pericentrally rotated structures (inverses) of isometric and isomorphs becomes: 


$$
f:\left[\begin{array}{ccc}
A=\left(x^{3}, x^{4}, x^{2}\right) & \delta & B=\left(x^{2}, x^{4}, x^{3}\right) \\
\Downarrow & \stackrel{\#}{*} & \Downarrow \\
R=(1,2,3) & 4 & (5,6,7)
\end{array}\right]
$$

Such that $A$ and $B$ are isometric isomorphs on tail-to-tail (its alternate) reflection about a centre $(\delta)$.

Then,

$$
\begin{gathered}
K_{c}=\frac{4\left(x^{3}+x^{4}+x^{2}+\delta+x^{2}+x^{4}+x^{3}\right)}{x^{3}+2 x^{4}+3 x^{2}+4 \delta+5 x^{2}+6 x^{4}+7 x^{3}} \\
K_{c}=\frac{4 x^{3}+4 x^{4}+4 x^{2}+4 \delta+4 x^{2}+4 x^{4}+4 x^{3}}{x^{3}+2 x^{4}+3 x^{2}+4 \delta+5 x^{2}+6 x^{4}+7 x^{3}} \\
K_{c}=\frac{8 x^{3}+8 x^{4}+8 x^{2}+4 \delta}{8 x^{3}+8 x^{4}+8 x^{2}+4 \delta}=1
\end{gathered}
$$

Therefore, $A$ and $B$ are invariant under pericentral rotation.

\section{Prove 2.5: Invariance under optical scaling}

Let the optinalytic construction of prove 3 be considered, and let $R+a$ be the change in scaling patterns. The optinalytic construction becomes:

$$
f:\left[\begin{array}{ccc}
A=\left(x^{2}, x^{4}, x^{3}\right) & \delta & B=\left(x^{3}, x^{4}, x^{2}\right) \\
\Downarrow & + & \Downarrow \\
R=[(1+a),(2+a),(3+a)] & (4+a) & {[(5+a),(6+a),(7+a)]}
\end{array}\right]
$$

Such that $A$ and $B$ are isometric isomorphs on head-to-head reflection about a centre $(\delta)$.

Then,

$$
\begin{gathered}
K_{c}=\frac{(4+a) \times\left(x^{2}+x^{4}+x^{3}+\delta+x^{3}+x^{4}+x^{2}\right)}{x^{2}(1+a)+x^{4}(2+a)+x^{3}(3+a)+\delta(4+a)+x^{3}(5+a)+x^{4}(6+a)+x^{2}(7+a)} \\
K_{c}=\frac{4 x^{2}+4 x^{4}+4 x^{3}+4 \delta+4 x^{3}+4 x^{4}+4 x^{2}}{x^{2}+2 x^{4}+3 x^{3}+4 \delta+5 x^{3}+6 x^{4}+7 x^{2}} \\
K_{c}=\frac{x^{2}(8+2 a)+x^{4}(8+2 a)+x^{3}(8+2 a)+\delta(4+a)}{x^{2}(8+2 a)+x^{4}(8+2 a)+x^{3}(8+2 a)+\delta(4+a)}=1
\end{gathered}
$$

Therefore, $A$ and $B$ are invariant under optical scaling.

\section{Prove 2.6: Invariance under central modulation}

Let the optinalytic construction of prove 3 be considered, and let $\delta \pm \beta$ be the central modulation, such that $\beta \in \mathbb{R}$. The optinalytic construction becomes:

$$
f:\left[\begin{array}{ccc}
A=\left(x^{2}, x^{4}, x^{3}\right) & \delta \pm \beta & B=\left(x^{3}, x^{4}, x^{2}\right) \\
\Downarrow & \stackrel{\leftrightarrow}{*} & \Downarrow \\
R=(1,2,3) & 4 & (5,6,7)
\end{array}\right]
$$


Such that $A$ and $B$ are isometric isomorphs on head-to-head reflection about a centre $(\delta)$.

Then,

$$
\begin{gathered}
K_{c}=\frac{4\left(x^{2}+x^{4}+x^{3}+(\delta \pm \beta) x^{3}+x^{4}+x^{2}\right)}{x^{2}+2 x^{4}+3 x^{3}+4(\boldsymbol{\delta} \pm \boldsymbol{\beta})+5 x^{3}+6 x^{4}+7 x^{2}} \\
K_{c}=\frac{4 x^{2}+4 x^{4}+4 x^{3}+4(\delta \pm \beta)+4 x^{3}+4 x^{4}+4 x^{2}}{x^{2}+2 x^{4}+3 x^{3}+4(\delta \pm \beta)+5 x^{3}+6 x^{4}+7 x^{2}} \\
K_{c}=\frac{8 x^{2}+8 x^{3}+8 x^{4}+4(\delta \pm \beta)}{8 x^{2}+8 x^{3}+8 x^{4}+4(\delta \pm \beta)}=1
\end{gathered}
$$

Therefore, $A$ and $B$ are invariant under central modulation.

\section{Theorem 3: Optinalytic invariance under operations (II)}

Asymmetrical or dissimilar state between isometric and isomorphic or automorphic remains invariant (the same) under product translation, central rotation (inversion), and optical scaling.

\section{Prove 3:}

Suppose we have an optinalytic construction of isometric and isomorphic spaces, sets and graphs with an assigned optical scale $(\mathrm{R}=-1,-2,-3,-4,-5,-6,-7)$ as follows:

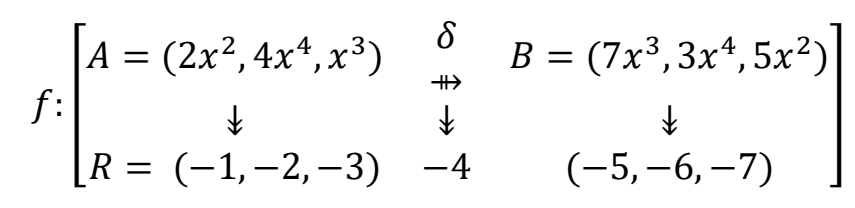

Such that $A$ and $B$ are isometric isomorphs on head-to-head reflection about a centre $(\delta)$.

Then,

$$
\begin{gathered}
K_{c}=\frac{-4\left(2 x^{2}+4 x^{4}+x^{3}+\delta+7 x^{3}+3 x^{4}+5 x^{2}\right)}{-2 x^{2}-8 x^{4}-3 x^{3}-4 \delta-35 x^{3}-18 x^{4}-35 x^{2}} \\
K_{c}=\frac{-8 x^{2}-16 x^{4}-4 x^{3}-4 \delta-28 x^{3}-12 x^{4}-20 x^{2}}{-2 x^{2}-8 x^{4}-3 x^{3}-4 \delta-35 x^{3}-18 x^{4}-35 x^{2}} \\
K_{c}=\frac{\left(-28 x^{2}-28 x^{4}-32 x^{3}\right)-4 \delta}{\left(37 x^{2}-26 x^{4}-38 x^{3}\right)-4 \delta} \\
K_{c}=\frac{-88-4 \delta}{-101-4 \delta} \neq 1
\end{gathered}
$$

Therefore, $A$ and $B$ are dissimilar (asymmetrical).

\section{Prove 3.1: Invariance under product translation}

Let the optinalytic construction of prove 4 be considered, and let ' $a$ ' be a translation factor. The optinalytic construction becomes: 


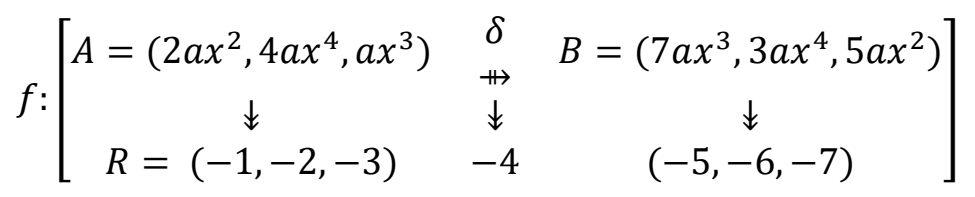

Such that $A$ and $B$ are isometric isomorphs on head-to-head reflection about a centre $(\delta)$.

Then,

$$
\begin{gathered}
K_{c}=\frac{-4\left(2 a x^{2}+4 a x^{4}+a x^{3}+\delta+7 a x^{3}+3 a x^{4}+5 a x^{2}\right)}{-2 x^{2}-8 x^{4}-3 x^{3}-4 \delta-35 x^{3}-18 x^{4}-35 x^{2}} \\
K_{c}=\frac{-8 a x^{2}-16 a x^{4}-4 a x^{3}-4 \delta-28 a x^{3}-12 a x^{4}-20 a x^{2}}{-2 a x^{2}-8 a x^{4}-3 a x^{3}-4 \delta-35 a x^{3}-18 a x^{4}-35 a x^{2}} \\
K_{c}=\frac{\left(-28 a x^{2}-28 a x^{4}-32 a x^{3}\right)-4 \delta}{\left(37 a x^{2}-26 a x^{4}-38 a x^{3}\right)-4 \delta} \\
K_{c}=\frac{-88-4 \delta}{-101-4 \delta} \neq 1
\end{gathered}
$$

Therefore, $A$ and $B$ are invariant under product translation.

\section{Prove 3.2: Invariance under central rotation (Inversion)}

Let the optinalytic construction of prove 4 be considered, the centrally rotated structures of isometric isomorphs become:

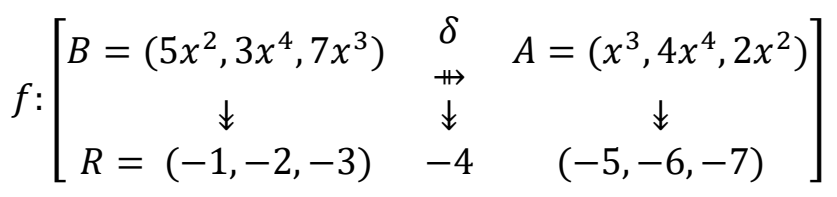

Such that $A$ and $B$ are isometric isomorphs on head-to-head reflection about a centre $(\delta)$.

Then,

$$
\begin{gathered}
K_{c}=\frac{-4\left(5 x^{2}+3 x^{4}+7 x^{3}+\delta+x^{3}+4 x^{4}+2 x^{2}\right)}{-5 x^{2}-6 x^{4}-21 x^{3}-4 \delta-5 x^{3}-24 x^{4}-14 x^{2}} \\
K_{c}=\frac{-20 x^{2}-12 x^{4}-28 x^{3}-4 \delta-4 x^{3}-16 x^{4}-8 x^{2}}{-5 x^{2}-6 x^{4}-21 x^{3}-4 \delta-5 x^{3}-24 x^{4}-14 x^{2}} \\
K_{c}=\frac{\left(-28 x^{3}-28 x^{4}-32 x^{2}\right)-4 \delta}{\left(-19 x^{3}-30 x^{4}-26 x^{2}\right)-4 \delta} \\
K_{c}=\frac{-88-4 \delta}{-75-4 \delta} \neq 1
\end{gathered}
$$

Therefore, $A$ and $B$ are invariant under product translation. 


\section{Prove 3.3: Invariance under optical scaling}

Let the optinalytic construction of prove 4 be considered, and let $(-R-a)$ be the change in scaling patterns. The optinalytic construction becomes:

$$
f:\left[\begin{array}{ccc}
A=\left(2 x^{2}, 4 x^{4}, x^{3}\right) & \delta & B=\left(7 x^{3}, 3 x^{4}, 5 x^{2}\right) \\
\Downarrow & \stackrel{\leftrightarrow}{\Downarrow} & \Downarrow \\
R=[(-1-a),(-2-a),(-3-a)] & (-4-a) & {[(-5-a),(-6-a),(-7-a)]}
\end{array}\right]
$$

Such that $A$ and $B$ are isometric isomorphs on head-to-head reflection about a centre $(\delta)$.

Then,

$$
\begin{gathered}
K_{c}=\frac{(-4-a) \times\left(2 x^{2}+4 x^{4}+x^{3}+\delta+7 x^{3}+3 x^{4}+5 x^{2}\right)}{2 x^{2}(-1-a)+4 x^{4}(-2-a)+x^{3}(-3-a)+\delta(-4-a)+} \\
7 x^{3}(-5-a)+3 x^{4}(-6-a)+5 x^{2}(-7-a) \\
K_{c}=\frac{\left(-28 x^{2}-2 a x^{2}\right)+\left(-16 x^{4}-4 a x^{4}\right)+\left(-4 x^{3}-a x^{3}\right)+(-4 \delta-a \delta)+}{\left(-2 x^{2}-2 a x^{2}\right)+\left(-8 x^{4}-4 a x^{4}\right)+\left(-3 a x^{4}\right)+\left(-20 x^{3}-5 a x^{2}\right)+(-4 \delta-a \delta)+} \\
K_{c}=\frac{\left(-35 x^{3}-7 a x^{3}\right)+\left(-18 x^{4}-3 a x^{4}\right)+\left(-35 x^{2}-5 a x^{2}\right)}{\left(37 x^{2}-7 a x^{2}\right)+\left(-26 x^{4}-7 a x^{4}\right)+\left(-38 x^{3}-7 a x^{3}\right)+(-4 \delta-a \delta)} \\
K_{c}=\frac{-88-(-4 \delta-a \delta)}{-101-(-4 \delta-a \delta)} \neq 1
\end{gathered}
$$

Therefore, $A$ and $B$ are invariant under optical scaling.

\section{Theorem 4: Optinalytic regulation}

An asymmetrical or dissimilar state between isometric and isomorphic or automorphic spaces, sets, and graphs can be transformed near-symmetrical or similar states under central modulation. A central modulation refers to the deliberate increase or decrease in quantity or function at the central midpoint $(\delta)$.

In other words, activating the central mid-node of an optinalytic construction between isometric isomorphs changes the size of the resultant fraction which in turn promotes the relationship (e.g symmetry, similarity, and identity). This is called central modulation or central normalization. The quantity or function used to change the relationship between isometric isomorphs is called normalization value $(\beta)$.

\section{Prove 4:}

Suppose we have an optinalytic construction as follows:

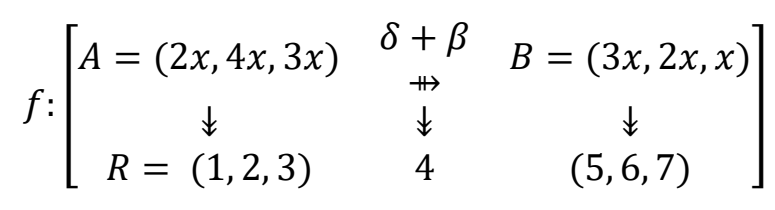


Such that $A$ and $B$ are isometric isomorphs on head-to-head reflection about a centre $(\delta)$.

Then,

$$
\begin{gathered}
K_{c}=\frac{4(2 x+4 x+3 x+(\delta+\beta)+3 x+2 x+x)}{2 x+8 x+9 x+4(\delta+\beta)+15 x+12 x+7 x} \\
K_{c}=\frac{8 x+16 x+12 x+4(\delta+\beta)+12 x+8 x+4 x}{2 x+8 x+9 x+4(\delta+\beta)+15 x+12 x+7 x} \\
K_{c}=\frac{60 x+4(\delta+\beta)}{53 x+4(\delta+\beta)}
\end{gathered}
$$

Thus,

$$
K_{c}=\frac{60 x}{53 x}+\frac{4(\delta+\beta)}{4(\delta+\beta)} \neq 1
$$

Therefore, $A$ and $B$ are not perfectly symmetrical, similar, and identical.

Let $\beta= \pm 1000$ such that $\delta \pm \beta= \pm 1000$

$$
\begin{gathered}
K_{c}=\frac{60 x}{53 x}+\frac{4 \times( \pm 1000)}{4 \times( \pm 1000)} \\
K_{c}=\frac{ \pm 4060 x}{ \pm 4053 x} \cong 1
\end{gathered}
$$

Therefore, $A$ and $B$ are normalized (modulated) similar (symmetrical).

\section{Theorem 5: Coefficient-percentage (C-P) translation models}

Let number $(x)$ be a finite subset of natural or real numbers such that $(x)$ range from -100 to 100. The percentage (probability) level of isometric isomorphism (similarity and identity) and isometric automorphism (symmetry) between a number $(x)$ and 100 is the number (x) itself if and only if the central node (mid-node) is not normalized (i.e $\delta=0$ ).

\section{Prove 6:}

We express the optinalytic construction of the theorem as:

$$
f:\left[\begin{array}{rcc}
x & \delta=0 & 100 \\
\Downarrow & \Downarrow & \Downarrow \\
R=1 & 2 & 3
\end{array}\right]
$$

Or the optinalytic construction is inversely expressed as:

$$
f:\left[\begin{array}{rcc}
100 & \delta=0 & x \\
\Downarrow & \stackrel{\#}{ } & \Downarrow \\
R=1 & 2 & 3
\end{array}\right]
$$

Then, Kabirian coefficient $\left(K_{C}\right)$ is defined as: 


$$
K_{c}=\frac{2(x+100)}{x+300}
$$

Or the Kabirian coefficient $\left(K_{c}\right)$ is inversely defined as:

$$
K_{c}=\frac{2(100+x)}{100+3 x}
$$

From the theorem, $x$ is defined as the percentage (probability) of the relationship between $x$ and 100 , then $x=\%$.

By making $x$ the subject of the formula, we obtain a model (Equation 3 and Figure 2) that intertranslates between Kabirian coefficient and percentages (probabilities):

$$
\%=x=\frac{200-300 K_{c}}{K_{c}-2}, \forall 0 \leq K_{c} \leq 1
$$

Or inversely as (Equation 4 and Figure 3):

$$
\%=x=\frac{200-100 K_{c}}{3 K_{c}-2}, \forall 1 \leq K_{c} \leq 2, \forall K_{c}<0, \& \forall K_{c}>2
$$

Translation of Kabirian coefficient is valid within a percentile (probability) range if and only if the outcomes are within range of values -100 to 100 (or -1 to 1 of its equivalent fractions).

The models that inter-convert or inter- translate Kabirian coefficients to percentages (probabilities) and vice versa are referred to as CP-distribution. The Figures 2-3 graphically presents the models of CP-distribution.

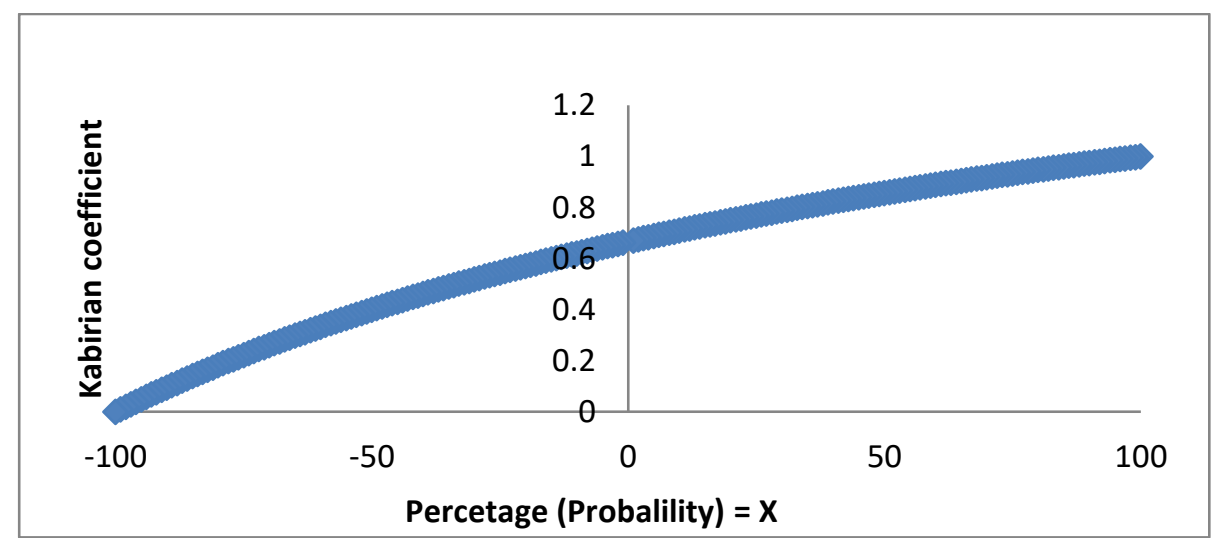

Figure 2: A modeled pattern of Kabirian coefficient within a percentile (probability) range. 


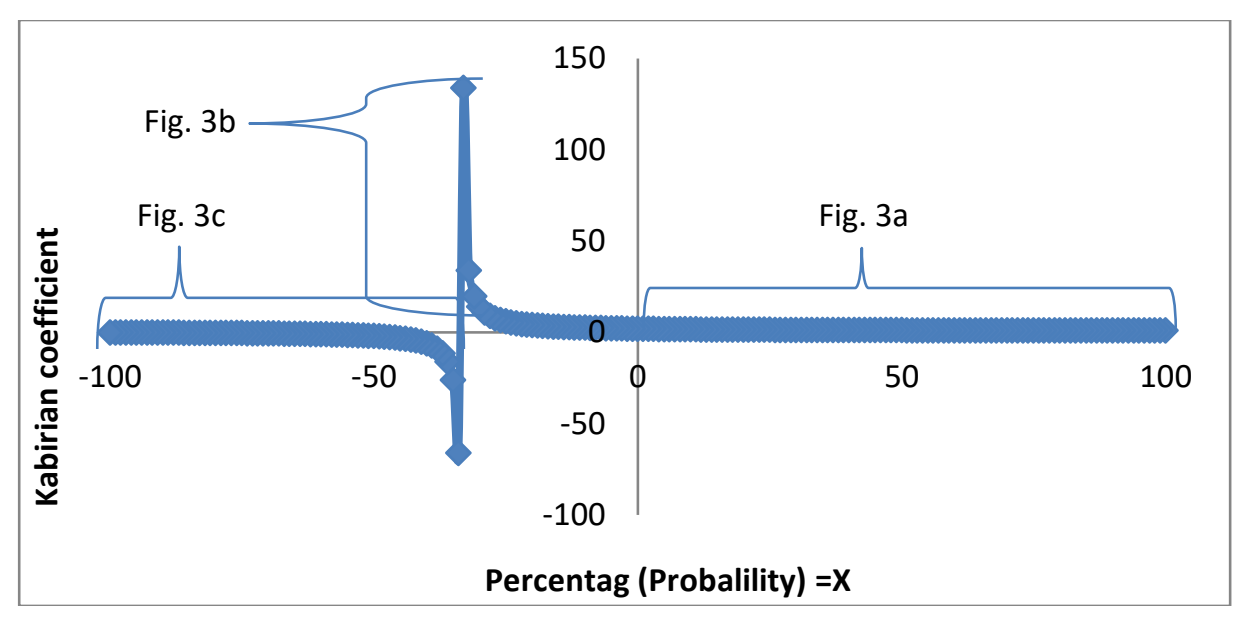

Figure 3: A modeled pattern of Kabirian coefficient within a percentile (probability) range.

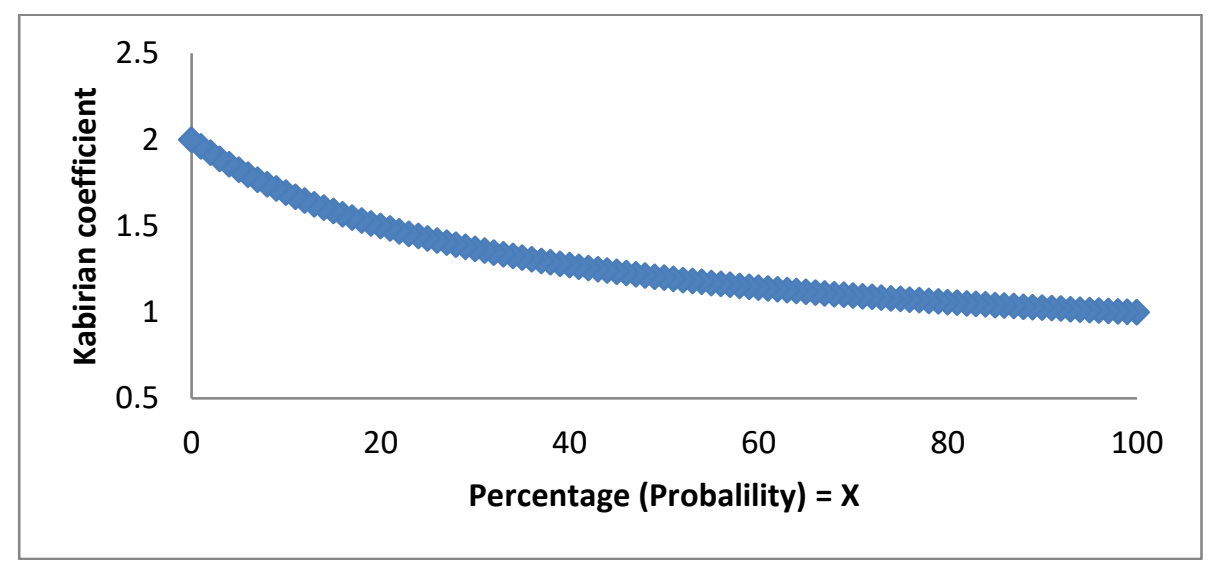

Figure 3a: A magnified part view showing the modeled pattern of Kabirian coefficient within a percentile (probability) range.

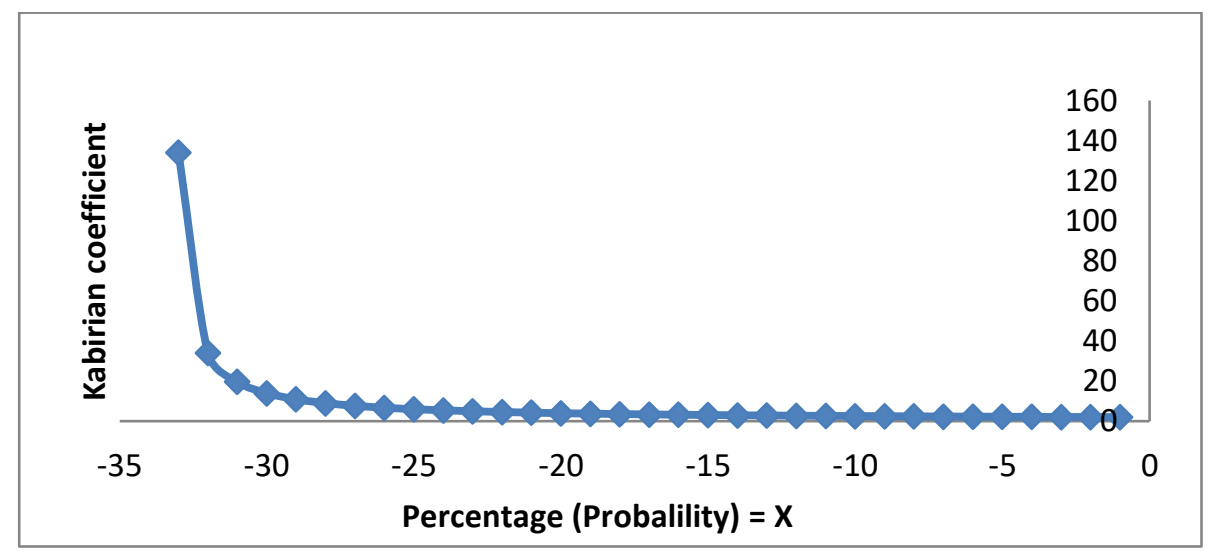

Figure 3b: A magnified part view showing the modeled pattern of the Kabirian coefficient within a percentile (probability) range. 


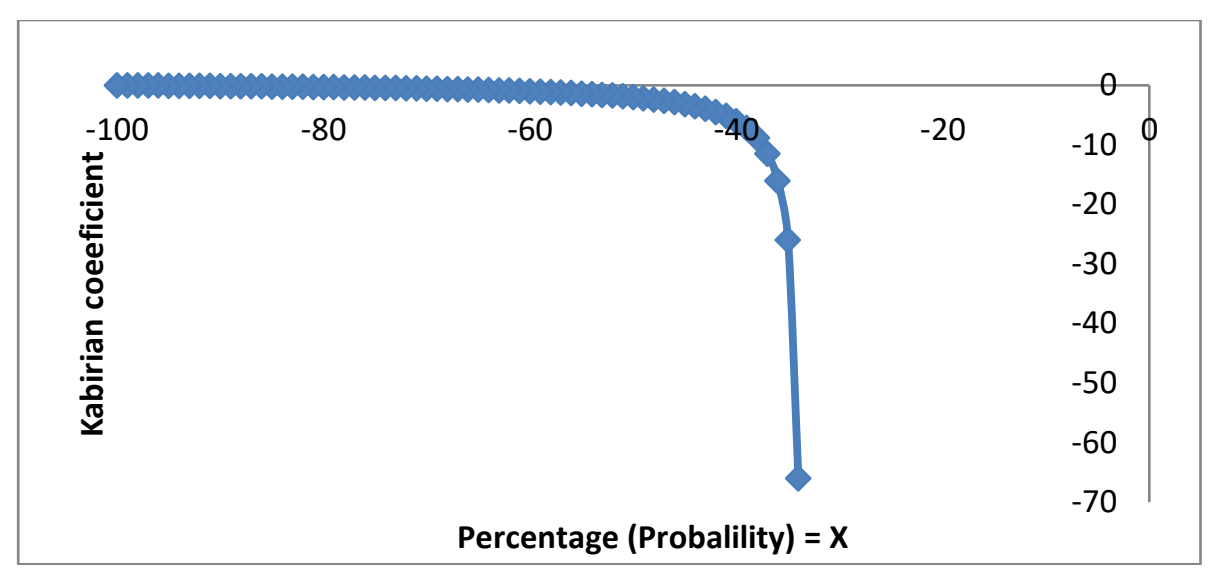

Figure 3c: A magnified part view showing the modeled pattern of the Kabirian coefficient within a percentile (probability) range.

\section{Theorem 6: Optinalytic inverse equivalence}

The Kabirian coefficients of optinalysis for inverse isometric isomorphs or automorphs are not equaled the same but have equal translated percentage (probability).

$$
\begin{aligned}
& K_{C}:\left(A{ }_{m}^{\delta} B \rightarrow R\right) \neq K_{C}:\left(B{ }_{\boxplus}^{\delta} A \rightarrow R\right)
\end{aligned}
$$

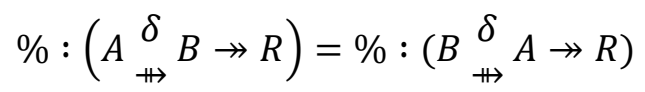

\section{Prove 6:}

Suppose we have an optinalytic construction as follows:

$$
f:\left[\begin{array}{ccc}
A=(2 x, 3 x, 6 x) & \delta=0 & B=(5 x, 3 x, x) \\
\Downarrow & \dddot{m} & \Downarrow \\
R=(1,2,3) & 4 & (5,6,7)
\end{array}\right]
$$

Such that $A$ and $B$ are isometric isomorphs or automorphs on head-to-head reflection about a centre $(\delta)$.

Then,

$$
\begin{gathered}
K_{c}=\frac{4(2 x+3 x+6 x+5 x+3 x+x)}{2 x+6 x+18 x+25 x+18 x+7 x} \\
K_{c}=\frac{8 x+12 x+24 x+20 x+12 x+4 x}{2 x+6 x+18 x+25 x+18 x+7 x} \\
K_{c}=\frac{80 x}{76 x}=\frac{20 x}{19 x}
\end{gathered}
$$

Inversely, the optinalytic construction becomes: 


$$
f:\left[\begin{array}{ccc}
B=(x, 3 x, 5 x) & \delta=0 & A=(6 x, 3 x, 2 x) \\
\Downarrow & \dddot{m} & \Downarrow \\
R=(1,2,3) & 4 & (5,6,7)
\end{array}\right]
$$

Such that $A$ and $B$ are isometric isomorphs or automorphs on tail-to-tail reflection about a centre $(\delta)$.

Then,

$$
\begin{gathered}
K_{c}=\frac{4(x+3 x+5 x+6 x+3 x+2 x)}{x+6 x+15 x+30 x+18 x+14 x} \\
K_{c}=\frac{4 x+12 x+20 x+24 x+12 x+8 x}{x+6 x+15 x+30 x+18 x+14 x} \\
K_{c}=\frac{80 x}{84 x}=\frac{20 x}{21 x}
\end{gathered}
$$

Now, the inverse equivalence by Kabirian coefficient is

$$
K_{C}:\left(A_{\Pi}^{\delta} B \rightarrow R\right) \neq K_{c}:\left(B_{\Pi \rightarrow}^{\delta} A \rightarrow R\right) \Rightarrow \frac{20 x}{19 x} \neq \frac{20 x}{21 x}
$$

Therefore, the inverse equivalences by the Kabirian coefficient do not equal to each other.

The inverse equivalence is as a result of the change (increase or decrease) in the denominator (i.e, the sum of all scalements of the existed nodes) by a certain factor called the coefficient of inverses.

Moreover, the valid translation of Kabirian coefficient to a percentage (probability):

For $K_{c}=\frac{20 x}{19 x}=1.052632$, by substituting it in a suitable equation $\left(\%=\frac{200-100 K_{c}}{3 K_{c}-2}\right)$, we have

$$
\%:\left(A_{\Pi}^{\delta} B \rightarrow R\right)=\frac{200-100 \times 1.052632}{3 \times 1.052632-2}=81.82
$$

Or inversely as:

For $K_{c}=\frac{20 x}{21 x}=0.952381$, by substituting it in a suitable equation $\left(\%=\frac{200-300 K_{c}}{K_{c}-2}\right)$, we have

$$
\%:(B \underset{\rightarrow}{\delta} A \rightarrow R)=\frac{200-300 \times 0.952381}{0.952381-2}=81.82
$$

Now, the inverse equivalence by percentage (probability) is

$$
\%:\left(A_{\rightarrow}^{\delta} B \rightarrow R\right)=\%:(B \underset{H}{\delta} A \rightarrow R) \Rightarrow 81.82=81.82
$$

Therefore, the inverse equivalences by percentage (probability) are equals to each other.

\section{Theorem 7: Deterministic optinalysis}

Similarity, identity, and symmetry of isometric isomorphs and automorphs from named spaces, sets, and graphs are deterministic by Kabirian coefficient on polynomial and non-polynomial graph models, and also by the translated percentages or probabilities on polynomial and non-polynomial graph models. 


\section{Prove 7:}

Definition 7.1: Iso-polymorphism: is the existence of an object or mathematical structures in many different forms in such a way that its structures and distances are preserved. In other words, isopolymorphism is a polymorphism that preserves the isometry and isomorphism of a mathematical object.

Definition 7.2: Single vertex iso-polymorphism: is an iso-polymorphism as a result of single vertex variation.

Suppose we have a graph $A$ and its iso-polymorphic variants $B_{1 \rightarrow N_{d}}$ from a named space, or sets. We can generate the iso-polymorphic variants by additive paranodic skewization with a skewization value $\geq t \infty$ over some generations (Abdullahi, 2019).

$$
A=\left(6 x, 3 x, 5 x, 9 x, 12 x, 24 x, \ldots \ldots N_{d}\right)
$$

The single vertex iso-polymorphic variants are:

$$
\begin{aligned}
& \mathrm{I}^{\text {st }} \text { generation }=B_{1}=\left(6 x+\boldsymbol{t}, 3 x, 5 x, 9 x, 12 x, 24 x, \ldots \ldots N_{d}\right) \\
& 2^{\text {nd }} \text { generation }=B_{2}=\left(6 x, 3 x+\boldsymbol{t}, 5 x, 9 x, 12 x, 24 x, \ldots \ldots N_{d}\right) \\
& 3^{\text {rd }} \text { generation }=B_{3}=\left(6 x, 3 x, 5 x+\boldsymbol{t}, 9 x, 12 x, 24 x, \ldots \ldots N_{d}\right) \\
& 4^{\text {th }} \text { generation }=B_{4}=\left(6 x, 3 x, 5 x, 9 x+\boldsymbol{t}, 12 x, 24 x, \ldots \ldots N_{d}\right) \\
& 5^{\text {th }} \text { generation }=B_{5}=\left(6 x, 3 x, 5 x, 9 x, 12 x+\boldsymbol{t}, 24 x, \ldots \ldots N_{d}\right) \\
& 6^{\text {th }} \text { generation }=B_{6}=\left(6 x, 3 x, 5 x, 9 x, 12 x, 24 x+\boldsymbol{t}, \ldots \ldots N_{d}\right)
\end{aligned}
$$

We may have an optinalytic construction as follows:

$$
f: \begin{array}{ccc}
\delta & =0 \\
& \rightarrow & B_{1 \rightarrow N_{d}}
\end{array} \rightarrow R
$$

And the inverse operation becomes:

$$
f: B_{1 \rightarrow N_{d}} \stackrel{\substack{=\\ \mathrm{H}}}{\mathrm{A}} \quad A_{1} \rightarrow R
$$

Then, the Kabirian coefficient between the graph $A_{1}$ and its iso-polymorphs $B_{1 \rightarrow N_{d}}$ are obtained as $Q$ and $S$ as valid translations (in percentage or probability) of $Q$ on C-P distributions.

Let $G$ be a set of positive integers ranks the iso-polymorphic generations (from the first to the last) of iso-polymorphs established.

By plotting regression graphs of $G$ against $Q$, we observe a moving and changing regression patterns (from the best fits of linear/simple polynomial/exponential, continues as simple polynomial, shifts to logarithmic, and finally stagnates at power) as a skewization value $a$ approaches a certain maximum (See Fig. 2). For further clarification, check Abdullahi (2019) at doi: 10.20944/preprints201911.0141.v1

However, by plotting regression graphs of $G$ against $Q$ of the inverse cases, we observe a moving and changing regression patterns (from the best fits of linear/simple polynomial/exponential, continues as simple polynomial, and finally stagnates at complex polynomial) as a skewization value $a$ approaches a 
certain maximum (See Fig. 3). For further clarification, check Abdullahi (2019) at doi: 10.20944/preprints201911.0141.v1

By plotting regression graphs of $G$ against $S$, we observe a moving and changing regression patterns (the best fits of from linear/simple polynomial/exponential, and finally stagnates at complex polynomials) as a skewization value $a$ approaches a certain maximum (See Fig. 4). Likewise, the explanations are the same for the inverse cases.

\section{Example Problem}

Suppose we have a graph $A$ with a nodality of 20 vertices, and its single vertex iso-polymorphs $B$ were generated using an additive paranodic skewization approach, with a skewization value $t=$ $10,25,50,10,10^{2}, \ldots \ldots 10^{10}$. The Kabirian coefficients and its valid translations were plotted graphically against its skewization value $t$ respectively.

$A_{(1-20)}=(25,28,15,5,55,10,33,12,70,90,45,64,23,87,67,34,97,45,34,78)$.

Let the optinalytic construction be defined as:

$$
f: A_{(1 \rightarrow 20)} \stackrel{\delta=0}{\rightarrow} \quad B_{(20 \rightarrow 1)} \rightarrow R=(1,2, \ldots \ldots, 41)
$$

The optinalysis of these datasets was carried out using a customized Excel sheets presented in the supplementary material attached to this article.

\section{Results}

The results present in Figures 4-6 reflect and justify theorem 7 and its explanations.

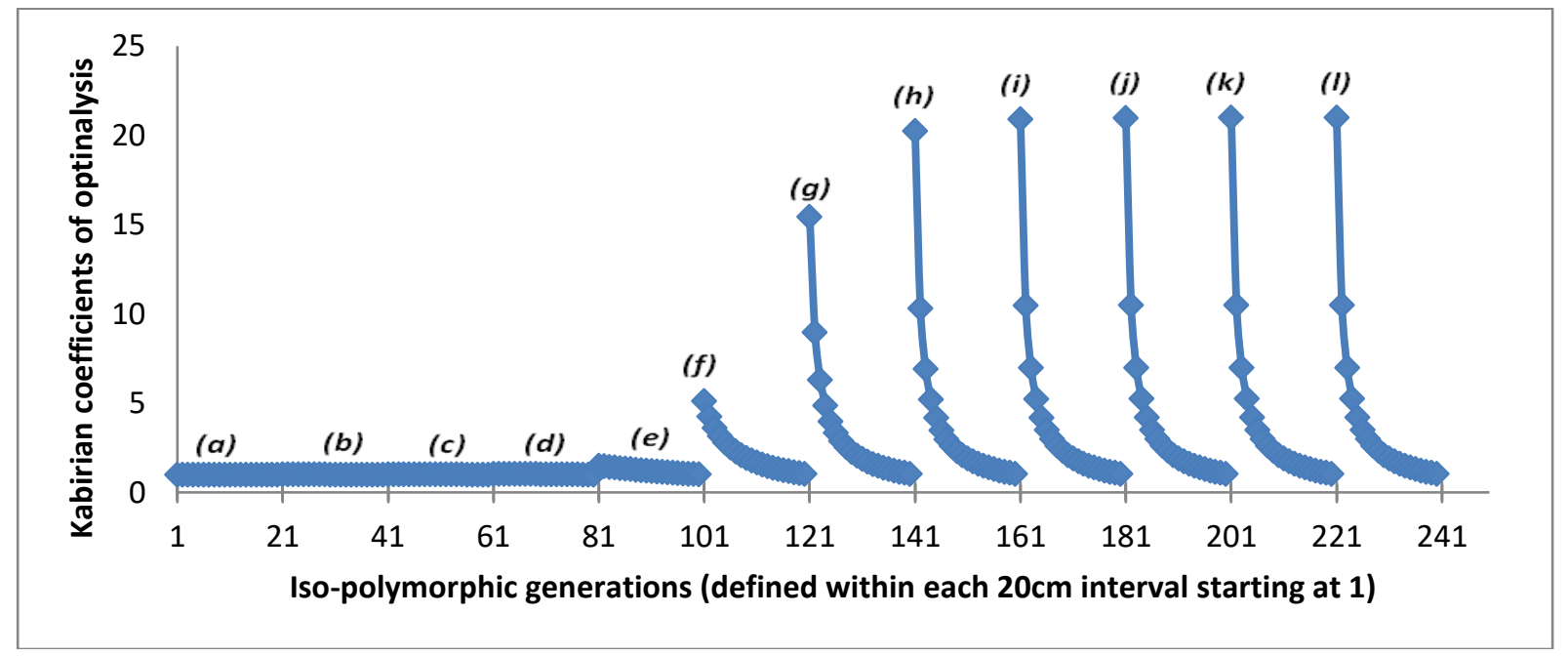

Figure 4: Graph models of the relationship between the generation number of iso-polymorphs and the resultant Kabirian coefficient of optinalysis. $(\boldsymbol{a})$ - $(\boldsymbol{l})$ are the graph models at skewization value $t=$ $10,25,50,10,10^{2}, \ldots \ldots 10^{10}$ respectively. $(\boldsymbol{a})$-(d) are linear/simple polynomial/exponential, $(\boldsymbol{e})$ is simple polynomial, $(\boldsymbol{f})$ is logarithmic, and $(\boldsymbol{g})$-( $(\boldsymbol{l})$ are power models.

Note: In this problem, the trending $K_{c}>1$ stagnates at a magnitude equal to the median optical scale. 


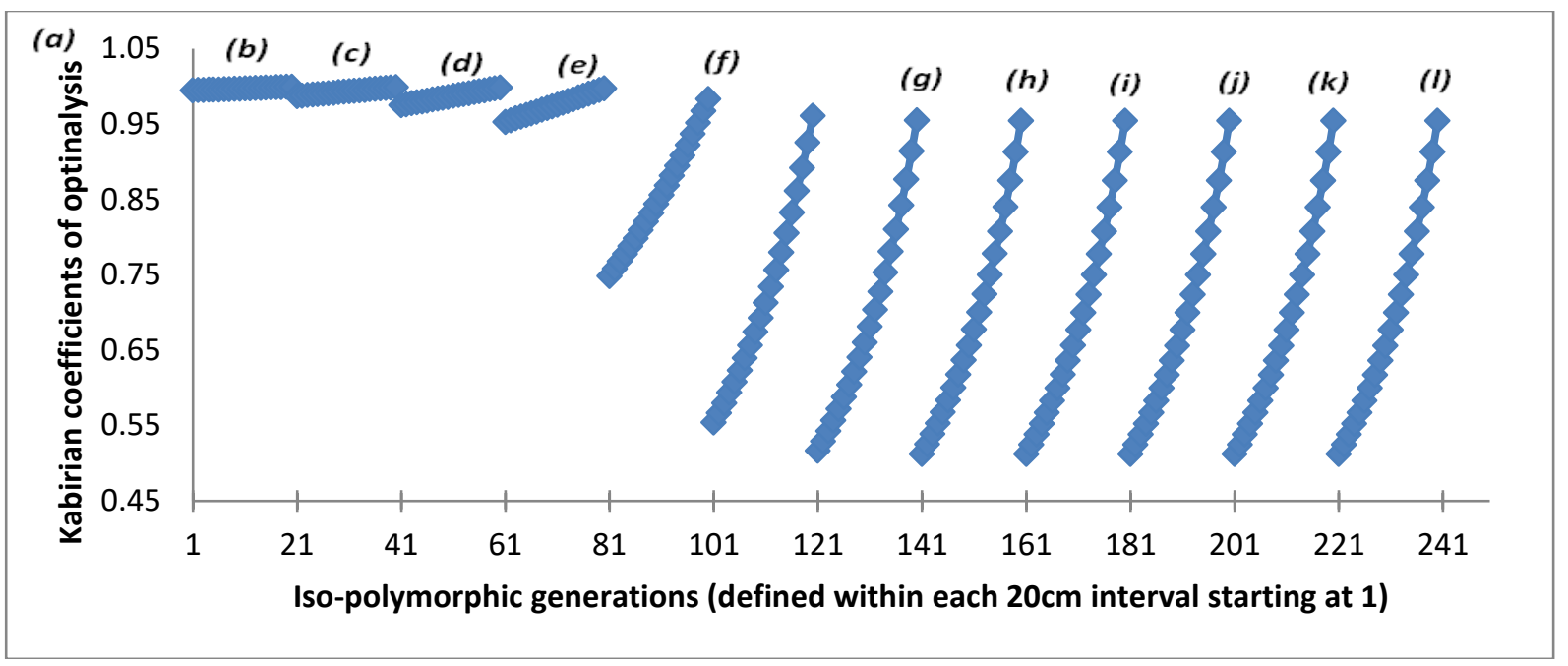

Figure 5: Graph models of the relationship between the generation number of iso-polymorphs and the resultant Kabirian coefficient of optinalysis of the inverse cases. (a)-(l) are the graph models at skewization value $t=$ $10,25,50,10,10^{2}, \ldots \ldots 10^{10}$ respectively. $(\boldsymbol{a})-(\boldsymbol{d})$ are linear/simple polynomial/exponential, $(\boldsymbol{e})$ is simple polynomial, $(\boldsymbol{f})-(\boldsymbol{l})$ are complex polynomial models.

Note: In this problem, the trending $K_{c}<1$ stagnates at a magnitude close to $\frac{1}{2}$.

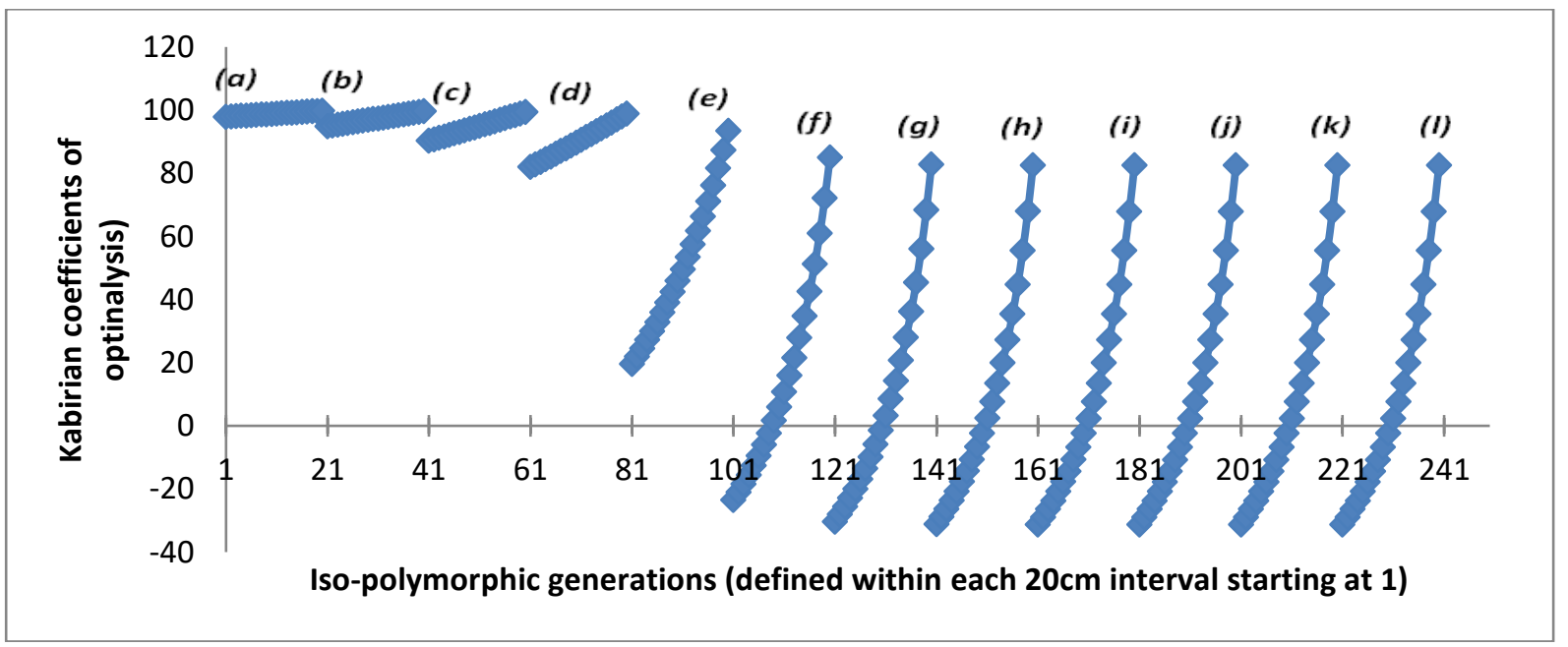

Figure 6: Graph models of the relationship between the generation number of iso-polymorphs and the translated percentages of optinalysis. $(\boldsymbol{a})$-(l) are the graph models at skewization value $t=10,25,50,10,10^{2}, \ldots \ldots 10^{10}$ respectively. $(\boldsymbol{a})-(\boldsymbol{d})$ are linear/simple polynomial/exponential, $(\boldsymbol{e})$ is simple polynomial, $(\boldsymbol{f})-(\boldsymbol{l})$ are complex polynomial models.

\section{Discussion}

In this paper, optinalysis represented an important paradigm of symmetry detections, similarity, and identity measures between two established isometric and isomorphic or automorphic graphs and sets. The stated theorems and the unique paradigm that governs it conforms to the concept and theorems of isometry and isomorphism. When we look at the optical scales, its uniform intervals preserve an equidistant relationship between the corresponding vertices or nodes of a named space, set, or graph. Furthermore, the optinalytic relationship between any pair of corresponding vertices is a clear bijection, 
which is also invertible, and these conform and translate the definitions and theorems of isometry, isomorphism, and automorphism.

When we look at the Kabirian coefficient function and its valid translation, we realize that the results remain the same (invariant) under mathematical operations or transformations. This is a preservation of the mathematical structures of the isomorphs or automorphs. However, these properties of optinalysis are sufficient evidence to prove its efficiency in graphs symmetry detection, graphs similarity, and identity measures.

Concerning methodological efficiency, the polynomial and non-polynomial graph models generated through optinalysis are very much analogous to the algorithmic formalization of polynomialtime of effectiveness of computational methods of graph isomorphism problem. Therefore, optinalysis is a complete solution to the graph isomorphism problems, unlike the quasi-polynomial algorithm proposed by Babai (Laszlo 2016).

\section{Conclusion}

Optinalysis is an efficient methodological paradigm proposed for symmetry detections, similarity, and identity measures between two established isometric and isomorphic or automorphic graphs and sets. The paradigm of optinalysis is based on isometry, isomorphism, and automorphism, expressed in clearly defined and proven theorems that conform to the definitions and theorems of isometry, isomorphism, and automorphism. Analogous to the polynomiality formalization of an efficient algorithm for graph isomorphism detections, optinalysis is however deterministic on polynomial and non-polynomial graph models.

Supplementary material: A supplementary file S1 is an Excel customized sheets for short range test in optinalysis. An example using a datasets were used to demonstrate the how it can be used.

\section{Conflict of interest}

The author declares no conflict of interest.

Funding: This research did not receive any specific grant from funding agencies in the public, commercial, or not-for-profit sectors.

\section{References}

Arora, Rashmi, and P Nigam, S. 2013. "A Comparative Study of Various Methods for Identification of Isomorphism in Kinematic Chains." In Proceedings of the 1st International and 16th National Conference on Machines and Mechanisms (INaCoMM2013), 582-89. IIT Roorkee, India,.

Hatori, Osamu, Takeshi Miura, and Hiroyuki Takagi. 2006. "Characterizations of Isometric Isomorphisms between Uniform Algebras via Nonlinear Range-Preserving Properties” 134 (10): 2923-30.

Laszlo, Babai. 2016. "Graph Isomorphism in Quasipolynomial Time.” In In Proceedings of the 48th Annual ACM SIGACT Symposium on Theory of Computing, 684-97. New York, NY, USA: ACM.

Mathon, Rudolf. 1979. "A Note on the Graph Isomorphism Counting Problem." Information Processing Letters 8 (3): 131-36.

Miillman, R, S, and D Parker, G. 1981. The Theory of Isometries. In: Geometry. Undergraduate Texts in Mathematics. New York, NY: Springer. https://doi.org/https://doi.org/10.1007/978-1-4684-01301_11. 
Oded, Goldreich, Micali Silvio, and Avi Wigderson. 1991. "Proofs That Yield Nothing but Their Validity or All Languages in Np Have Zero-Knowledge Proof Systems." Journal of the ACM (JACM) 38 (3): 690-728.

Ronald, C, Read, and Corneil Derek, G. 1977. "The Graph Isomorphism Disease.” Journal of Graph Theory 1 (4): 339-63.

Shane, Syed, Haider Rizvi, Ali Hasan, and D Ph. 2014. "A New Concept to Detect Isomorphism in Kinematic Chains Using Fuzzy Similarity Index" 86 (12): 30-33. 